\title{
Lie Bialgebra Structures for Centrally Extended Two-Dimensional Galilei Algebra and their Lie-Poisson Counterparts
}

\author{
Anna Opanowicz* \\ Department of Theoretical Physics II \\ University of Łódź \\ Pomorska 149/153, 90-236 Łódź, Poland
}

\begin{abstract}
All bialgebra structures for centrally extended Galilei algebra are classified. The corresponding Lie-Poisson structures on centrally extended Galilei group are found.
\end{abstract}

*Supported by Łódź University Grant No. 580 


\section{Introduction}

Much interest has been attracted in last years to the problem of deformations of space-time symmetry groups [1], [2], [3], [4], [5], [6], [6]. In particular, in the recent paper [8] all inequivalent bialgebra structures on two-dimensional Galilei algebra were classified and the corresponding Lie-Poisson structures on the group were found.

From the physical point of view what is really interesting is the central extension of the Galilei algebra. This is because only the genuine projective representations of the Galilei group are relevant in nonrelativistic quantum theory [9]. In the present paper we classify all nonequivalent bialgebra Lie-Poisson structures for centrally extended two-dimensional Galilei algebra/group. In the two-dimensional case there exists two-parameter family of central extensions, the parameters being the mass of the particle and the constant force acting on it. We restrict ourselves to the case of free particles, i.e. only the mass parameter is kept nonvanishing.

The content of the paper is as follows. First, we find the general form of 1cocycle on centrally extended twodimesional Galilei algebra. Then the action of most general automorphism transformation on such 1-cocycle is considered and its orbits are classified which allows to find all nonequivalent bialgebra structures. The corresponding Lie-Poisson structures on Galilei group are then found. The whole procedure follows quite closely the one presented in Ref. [10] for $E(2)$ group and in Ref. [8] for two-dimensional Galilei group. As a result we find 26 nonequivalent bialgebra structures (some of them being still one parameter families), 8 of them being the coboundary ones.

\section{Two-dimensional Galilei group and algebra with central extension and their automor- phisms}

The two-dimensional Galilei group is a Lie group of transformations of the space-time with one space dimensions. An arbitrary group element $g$ is of the form

$$
g=(\tau, v, a)
$$


here $\tau$ is time translation, $a$ respectively $v$ are space translation respectively Galilean boost. The multiplication law reads:

$$
g^{\prime} g=\left(\tau^{\prime}+\tau, v^{\prime}+v, a^{\prime}+a+\tau v^{\prime}\right)
$$

The resulting Lie algebra takes the form:

$$
[K, H]=i P, \quad[K, P]=0, \quad[H, P]=0
$$

The central extension is obtained by replacing the second commutation rule by

$$
[K, P]=i M
$$

where

$$
[M, \cdot]=0 .
$$

Therefore, we arrive finally at the following algebra

$$
[K, H]=i P, \quad[K, P]=i M, \quad[H, P]=0, \quad[M, \cdot]=0
$$

Let us define the centrally extended Galilei group by the following global expotential parametrization of group elements

$$
\widetilde{g}=e^{i m M} e^{-i \tau H} e^{i a P} e^{i v K}
$$

Let us write

$$
\widetilde{g}=(m, \tau, v, a)
$$

Then we have the following multiplication law

$$
\widetilde{g}^{\prime} \widetilde{g}=\left(m^{\prime}+m-\frac{1}{2} v^{\prime 2} \tau-a v^{\prime}, \tau^{\prime}+\tau, v^{\prime}+v, a^{\prime}+a+\tau v^{\prime}\right)
$$

Lie algebra with central extension can be realized in terms of right invariant fields to be calculated according to the standard rules from the composition law (9)

$$
\begin{aligned}
X_{v}^{R} & =i\left(\frac{\partial}{\partial v}-a \frac{\partial}{\partial m}+\tau \frac{\partial}{\partial a}\right) \\
X_{a}^{R} & =i \frac{\partial}{\partial a} \\
X_{m}^{R} & =i \frac{\partial}{\partial m} \\
X_{\tau}^{R} & =-i \frac{\partial}{\partial \tau}
\end{aligned}
$$


Let us now describe all automorphisms of the algebra (6). The group of automorphisms consists of the following transformations

$$
\left(\begin{array}{l}
K \\
H \\
P \\
M
\end{array}\right) \rightarrow\left(\begin{array}{l}
K^{\prime} \\
H^{\prime} \\
P^{\prime} \\
M^{\prime}
\end{array}\right)=\left(\begin{array}{cccc}
\gamma_{3} & \alpha_{3} & \beta_{3} & \eta_{3} \\
0 & \alpha_{1} & \beta_{1} & \eta_{1} \\
0 & 0 & \beta_{2} & \eta_{2} \\
0 & 0 & 0 & \eta_{4}
\end{array}\right)\left(\begin{array}{c}
K \\
H \\
P \\
M
\end{array}\right)
$$

where :

$$
\begin{aligned}
& \beta_{2}=\gamma_{3} \alpha_{1} \\
& \eta_{2}=\gamma_{3} \beta_{1} \\
& \eta_{4}=\gamma_{3} \beta_{2},
\end{aligned}
$$

and, obviously, $\alpha_{1} \neq 0, \gamma_{3} \neq 0$.

\section{The Bialgebra structures on two-dimen- sional centrally extended Galilei algebra}

Our aim here is to give a complete classification of Lie bialgebra structures for the algebra (6) up to automorphisms.

Let us remind the definition of bialgebra. It is a pair $(L, \delta)$, where $L$ is a Lie algebra while $\delta$ is a skewsymmetric cocommutator $\delta: L \rightarrow L \otimes L$, i.e.

(i) $\delta$ is a 1-cocycle,

$$
\delta([X, Y])=[\delta(X), 1 \otimes Y+Y \otimes 1]+[1 \otimes X+X \otimes 1, \delta(Y)] \text { for } \mathrm{X}, \mathrm{Y} \in \mathrm{L}
$$

(ii) The dual map $\delta^{*}: L^{*} \otimes L^{*} \rightarrow L^{*}$ defines a Lie bracket on $L^{*}$.

We can find all bialgebra structures on our algebra. The general form of $\delta$ obeying (i) is

$$
\left(\begin{array}{l}
\delta(H) \\
\delta(P) \\
\delta(K) \\
\delta(M)
\end{array}\right)=\left(\begin{array}{cccccc}
a & 0 & 0 & b & c & d \\
0 & 0 & 0 & a & 0 & h-b \\
e & 0 & f & g & h & j \\
0 & 0 & 0 & 0 & 0 & -(a+f)
\end{array}\right)\left(\begin{array}{c}
H \wedge P \\
H \wedge K \\
P \wedge K \\
H \wedge M \\
M \wedge K \\
M \wedge P
\end{array}\right),
$$


$\mathrm{a}, \mathrm{b}, \mathrm{c}, \mathrm{d}, \mathrm{e}, \mathrm{f}, \mathrm{g}, \mathrm{h}$ and $\mathrm{j}$ being arbitrary real parameters.

From the condition (ii) we obtain:

$$
a=b=c=0
$$

or

$$
a=e=f=0
$$

or

$$
b=c=f=h=0
$$

Eqs. (13) and (14) define all bialgebra structures on two-dimensional Galilei algebra (6). However, we are interested in classification of nonequivalent bialgebra structures. To this end we find the transformation rules for the parameters under the automorphisms (11). They read

$$
\begin{aligned}
& \widetilde{a}=\frac{a}{\beta_{2}} \\
& \widetilde{b}=\frac{b}{\eta_{4}}+\frac{\alpha_{3} \alpha_{1}}{\beta_{2} \eta_{4}} c \\
& \widetilde{c}=\frac{\alpha_{1}}{\eta_{4} \gamma_{3}} c \\
& \widetilde{d}=\frac{\alpha_{1}}{\beta_{2} \eta_{4}} d-2 \frac{\eta_{1} \alpha_{1}}{\beta_{2}{ }^{3}} a+\frac{\alpha_{1}\left(\alpha_{3} \beta_{1}-\alpha_{1} \beta_{3}\right)}{\beta_{2}{ }^{2} \eta_{4}} c+\frac{\beta_{1} \eta_{2}}{\beta_{2}{ }^{2} \eta_{4}} a+\frac{\beta_{1}}{\beta_{2} \eta_{4}} h-\frac{\eta_{1}}{\beta_{2} \eta_{4}} f \\
& \widetilde{e}=\frac{\gamma_{3}}{\alpha_{1} \beta_{2}} e+\frac{\alpha_{3} \gamma_{3}}{\beta_{2}{ }^{2}} f+\frac{\alpha_{3}}{\alpha_{1} \beta_{2}} a \\
& \widetilde{f}=\frac{f}{\beta_{2}} \\
& \widetilde{g}=\frac{\gamma_{3}}{\alpha_{1} \eta_{4}} g-\frac{\beta_{1} \gamma_{3}}{\alpha_{1} \beta_{2}{ }^{2}} e-\frac{\alpha_{3} \eta_{2}}{\beta_{2}{ }^{3}} f+\frac{\alpha_{3}}{\alpha_{1} \eta_{4}} b+\frac{\alpha_{3} \gamma_{3}}{\beta_{2} \eta_{4}} h-\frac{\beta_{1} \alpha_{3}}{\beta_{2}{ }^{2} \alpha_{1}} a+\frac{\alpha_{3}}{\beta_{2} \eta_{4}} c+\frac{\beta_{3}}{\alpha_{1} \eta_{4}} a \\
& \widetilde{j}=\frac{\gamma_{3}}{\beta_{2} \eta_{4}} j-\frac{\eta_{1} \gamma_{3}}{\beta_{2}{ }^{3}} e-\frac{\alpha_{3} \eta_{1}}{\beta_{2}{ }^{3}} f+\frac{\gamma_{3} \eta_{2}}{\beta_{2}{ }^{2} \eta_{4}} g+\frac{\alpha_{3} \beta_{1}}{\beta_{2}{ }^{3}} h-\frac{\alpha_{3} \eta_{1}}{\beta_{2}{ }^{3}} a+\frac{\alpha_{3} \eta_{2}}{\beta_{2}{ }^{2} \eta_{4}} b \\
& \\
& +\frac{\alpha_{3}\left(\alpha_{3} \beta_{1}-\alpha_{1} \beta_{3}\right)}{\beta_{2}{ }^{2} \eta_{4}} c+\frac{\alpha_{3}}{\beta_{2} \eta_{4}} d+\frac{\eta_{2} \beta_{3}}{\beta_{2}{ }^{2} \eta_{4}} a-\frac{\beta_{3}}{\beta_{2} \eta_{4}} b-\frac{\eta_{3}}{\beta_{2} \eta_{4}} a \\
& \widetilde{h}=\frac{h}{\eta_{4}}-\frac{\beta_{1}}{\beta_{2}{ }^{2}} f+\frac{\alpha_{3}}{\eta_{4} \gamma_{3}} c \\
& \widetilde{h}-\widetilde{b}=\frac{h-b}{\eta_{4}}-\frac{\eta_{2}}{\beta_{2} \eta_{4}} f \\
& \widetilde{a}+\widetilde{f}=\frac{a+f}{\beta_{2}}
\end{aligned}
$$

We are now in position to classify all orbits of automorphism group in the space of bialgebra structures. A simple but long and painful analysis leads to the complete list of Lie bialgebra structures summarized in Table 1. 
Table 1

\begin{tabular}{|c|c|c|c|c|c|c|c|c|c|c|}
\hline & $\mathrm{a}$ & $\mathrm{b}$ & $\mathrm{c}$ & $\mathrm{d}$ & $\mathrm{e}$ & $\mathrm{f}$ & $\mathrm{g}$ & $\mathrm{h}$ & $\mathrm{j}$ & Remarks \\
\hline \hline 1 & 0 & 0 & 0 & 0 & 1 & 0 & 0 & 0 & 0 & \\
\hline 2 & 0 & 0 & 0 & 0 & -1 & 0 & 0 & 0 & 0 & \\
\hline 3 & 0 & 0 & 0 & 0 & 0 & 0 & 0 & 1 & 0 & coboundary \\
\hline 4 & 0 & 0 & 0 & 0 & 0 & 0 & 1 & 0 & 0 & coboundary \\
\hline 5 & 0 & 0 & 0 & 1 & 0 & 0 & 0 & 0 & 0 & coboundary \\
\hline 6 & 0 & 0 & 0 & 0 & 0 & 0 & 0 & 0 & 1 & coboundary \\
\hline 7 & 0 & 0 & 0 & 0 & 0 & 0 & 0 & 0 & -1 & coboundary \\
\hline 8 & 0 & 0 & 0 & 0 & 0 & 0 & 0 & 1 & 1 & coboundary \\
\hline 9 & 0 & 0 & 0 & 0 & 0 & 0 & 0 & 1 & -1 & coboundary \\
\hline 10 & 0 & 0 & 0 & 0 & 1 & 0 & 0 & 1 & 0 & \\
\hline 11 & 0 & 0 & 0 & 0 & -1 & 0 & 0 & 1 & 0 & \\
\hline 12 & 0 & 0 & 0 & 1 & 0 & 0 & 1 & 0 & 0 & coboundary \\
\hline 13 & 0 & 0 & 0 & 0 & 0 & 1 & 0 & 0 & $\varepsilon$ & $\varepsilon \in \mathrm{R}$ \\
\hline 14 & 0 & 0 & 0 & 0 & 0 & 1 & 1 & 0 & $\varepsilon$ & $\varepsilon \in \mathrm{R}$ \\
\hline 15 & 0 & 0 & 0 & 1 & 1 & 0 & 0 & 0 & 0 & \\
\hline 16 & 0 & 0 & 0 & 1 & -1 & 0 & 0 & 0 & 0 & \\
\hline 17 & 0 & 1 & 0 & 0 & 0 & 0 & 0 & 0 & 0 & \\
\hline 18 & 0 & 1 & 0 & 1 & 0 & 0 & 0 & 0 & 0 & \\
\hline 19 & 0 & $\varepsilon$ & 0 & 0 & 0 & 0 & 0 & 1 & 0 & $\varepsilon \neq 0$ \\
\hline 20 & 0 & -1 & 0 & 0 & 0 & 0 & 1 & 1 & 0 & \\
\hline 21 & 0 & $\varepsilon$ & 1 & 0 & 0 & 0 & 0 & 0 & 0 & $\varepsilon \in \mathrm{R}$ \\
\hline 22 & 0 & $\varepsilon$ & 1 & 0 & 0 & 0 & 0 & 0 & 1 & $\varepsilon \in \mathrm{R}$ \\
\hline 23 & 0 & $\varepsilon$ & 1 & 0 & 0 & 0 & 0 & 0 & -1 & $\varepsilon \in \mathrm{R}$ \\
\hline 24 & 0 & $\varepsilon$ & 1 & 0 & 0 & 0 & 1 & 0 & 0 & $\varepsilon \in \mathrm{R}$ \\
\hline 25 & 0 & $\varepsilon$ & 1 & 0 & 0 & 0 & -1 & 0 & 0 & $\varepsilon \in \mathrm{R}$ \\
\hline 26 & 1 & 0 & 0 & 0 & 0 & 0 & 0 & 0 & 0 & \\
\hline & & & & & & & \\
\hline
\end{tabular}

We have checked explictly that all the above bialgebra structures are consistent and inequivalent. It remains to find coboundary structures (listed also in Table1).

As it is well known a cocommutator $\delta$ given by

$$
\delta(X)=i[1 \otimes X+X \otimes 1, r], r \in L \wedge L, X \in L
$$


defines a coboundary Lie bialgebra if and only if $r$ fulfills the modified classical Yang-Baxter equation

$$
[X \otimes 1 \otimes 1+1 \otimes X \otimes 1+1 \otimes 1 \otimes X, \xi(r)]=0, X \in L,
$$

where $\xi(r)$ is the Schouten bracket

$$
\xi(r) \equiv\left[r_{12}, r_{13}\right]+\left[r_{12}, r_{23}\right]+\left[r_{13}, r_{23}\right] ;
$$

here

$$
\begin{gathered}
r_{12}=r^{i j} X_{i} \otimes X_{j} \otimes 1 \\
r_{13}=r^{i j} X_{i} \otimes 1 \otimes X_{j} \\
r_{23}=r^{i j} 1 \otimes X_{i} \otimes X_{j} .
\end{gathered}
$$

Let us put

$$
r=A H \wedge P+C P \wedge K+D H \wedge M+E M \wedge K+F M \wedge P
$$

Egs. (18) and (16) give now

$$
\begin{aligned}
& \delta(P)=-C M \wedge P \\
& \delta(H)=E M \wedge P \\
& \delta(K)=-A H \wedge M-C M \wedge K+D M \wedge P \\
& \delta(M)=0
\end{aligned}
$$

By comparying Eqs. (13) and (19) we get

$$
\begin{aligned}
& a=b=c=e=f=0 \\
& A=-g, C=-h, D=j, E=d
\end{aligned}
$$

which serve to identify the coboundary structures in Table 1.

\section{The Lie-Poisson structures on two-dimen- sional Galilei group}

In this section we find all Lie-Poisson structures on centrally extended twodimensional Galilei group. Let $G$ be a Lie group , $L$ its Lie algebra and $\left\{X_{i}^{R}\right\}$ - the set of right invariant fields on $G$. As it is well known

$$
\{\Psi, \Phi\} \equiv \eta^{i j}(g) X_{i}^{R} \Psi X_{j}^{R} \Phi
$$


where

$$
\eta(g)=\eta^{i j}(g) X_{i} \otimes X_{j}, \eta: G \rightarrow \Lambda^{2} L
$$

provides $G$ with a Poisson-Lie group structure if only if

$$
\begin{aligned}
& \text { (i) } \eta^{i l} X_{l}^{R} \eta^{j k}+\eta^{k l} X_{l}^{R} \eta^{i j}+\eta^{j l} X_{l}^{R} \eta^{k i} \\
& \quad-c_{l p}^{j} \eta^{i l} \eta^{p k}-c_{l p}^{i} \eta^{k l} \eta^{p j}-c_{l p}^{k} \eta^{j l} \eta^{p i}=0 \\
& \text { (ii) } \eta\left(g^{\prime} g\right)=\eta\left(g^{\prime}\right)+A d g^{\prime} \eta(g)
\end{aligned}
$$

In our case let us define

$$
\begin{aligned}
\eta(m, \tau, a, v)= & \lambda(m, \tau, a, v) H \wedge P+\mu(m, \tau, a, v) H \wedge K \\
& +\nu(m, \tau, a, v) P \wedge K+\kappa(m, \tau, a, v) H \wedge M \\
& +\rho(m, \tau, a, v) M \wedge K+\pi(m, \tau, a, v) M \wedge P
\end{aligned}
$$

Eq. (23) gives

$$
\begin{aligned}
\eta\left(g^{\prime} g\right)= & \left(\lambda^{\prime}+\lambda-\tau^{\prime} \mu\right) H \wedge P+\left(\nu^{\prime}+\nu-v^{\prime} \mu\right) P \wedge K \\
& +\left(\kappa^{\prime}+\kappa-v^{\prime} \lambda+a^{\prime} \mu\right) H \wedge M+\left(\mu^{\prime}+\mu\right) H \wedge K \\
& +\left(\rho^{\prime}+\rho+\frac{v^{\prime 2}}{2} \mu-v^{\prime} \nu\right) M \wedge K \\
& +\left(\pi^{\prime}+\pi-\frac{v^{\prime 2}}{2} \lambda+\left(v^{\prime} a^{\prime}-\frac{v^{\prime 2} \tau^{\prime}}{2}\right) \mu+\left(v^{\prime} \tau^{\prime}-a^{\prime}\right) \nu+v^{\prime} \kappa-\tau^{\prime} \rho\right) M \wedge P
\end{aligned}
$$

where $\lambda^{\prime} \equiv \lambda\left(m^{\prime}, \tau^{\prime}, a^{\prime}, v^{\prime}\right)$ etc.

Consequently we obtain the following set of equations determining $\lambda, \mu$, $\nu, \kappa, \rho$ and $\pi$

$$
\begin{aligned}
\lambda\left(m^{\prime \prime}, \tau^{\prime \prime}, a^{\prime \prime}, v^{\prime \prime}\right)= & \lambda\left(m^{\prime}, \tau^{\prime}, a^{\prime}, v^{\prime}\right)+\lambda(m, \tau, a, v)-\tau^{\prime} \mu(m, \tau, a, v) \\
\mu\left(m^{\prime \prime}, \tau^{\prime \prime}, a^{\prime \prime}, v^{\prime \prime}\right)= & \mu\left(m^{\prime}, \tau^{\prime}, a^{\prime}, v^{\prime}\right)+\mu(m, \tau, a, v) \\
\nu\left(m^{\prime \prime}, \tau^{\prime \prime}, a^{\prime \prime}, v^{\prime \prime}\right)= & \nu\left(m^{\prime}, \tau^{\prime}, a^{\prime}, v^{\prime}\right)+\nu(m, \tau, a, v)-v^{\prime} \mu(m, \tau, a, v) \\
\kappa\left(m^{\prime \prime}, \tau^{\prime \prime}, a^{\prime \prime}, v^{\prime \prime}\right)= & \kappa\left(m^{\prime}, \tau^{\prime}, a^{\prime}, v^{\prime}\right)+\kappa(m, \tau, a, v)-v^{\prime} \lambda(m, \tau, a, v) \\
& +a^{\prime} \mu(m, \tau, a, v) \\
\rho\left(m^{\prime \prime}, \tau^{\prime \prime}, a^{\prime \prime}, v^{\prime \prime}\right)= & \rho\left(m^{\prime}, \tau^{\prime}, a^{\prime}, v^{\prime}\right)+\rho(m, \tau, a, v)+\frac{1}{2} v^{\prime 2} \mu(m, \tau, a, v) \\
& -v^{\prime} \nu(m, \tau, a, v)
\end{aligned}
$$




$$
\begin{aligned}
\pi\left(m^{\prime \prime}, \tau^{\prime \prime}, a^{\prime \prime}, v^{\prime \prime}\right)= & \pi\left(m^{\prime}, \tau^{\prime}, a^{\prime}, v^{\prime}\right)+\pi(m, \tau, a, v)-\frac{1}{2} v^{2} \lambda(m, \tau, a, v) \\
& +v^{\prime} \kappa(m, \tau, a, v)+\left(v^{\prime} a^{\prime}-\frac{1}{2} v^{\prime 2} \tau^{\prime}\right) \mu(m, \tau, a, v) \\
& +\left(v^{\prime} \tau^{\prime}-a^{\prime}\right) \nu(m, \tau, a, v)-\tau^{\prime} \rho(m, \tau, a, v)
\end{aligned}
$$

The strategy to solve Eq. (26) is to find first the form of $\eta$ for 1-parameter subgroups generated by $P, K, H, M$ and use again Eq. (26) together with the decomposition

$$
(m, \tau, a, v)=(m, 0,0,0) *(0, \tau, 0,0) *(0,0, a, 0) *(0,0,0, v)
$$

to determine the form of $\eta$ for general group element. Eq. (26) as specialized for one-parameter subgroups generated by $M, H, P$ and $K$ read

$$
\begin{aligned}
& \lambda\left(m^{\prime \prime}, 0,0,0\right)=\lambda\left(m^{\prime}, 0,0,0\right)+\lambda(m, 0,0,0) \\
& \mu\left(m^{\prime \prime}, 0,0,0\right)=\mu\left(m^{\prime}, 0,0,0\right)+\mu(m, 0,0,0) \\
& \nu\left(m^{\prime \prime}, 0,0,0\right)=\nu\left(m^{\prime}, 0,0,0\right)+\nu(m, 0,0,0) \\
& \kappa\left(m^{\prime \prime}, 0,0,0\right)=\kappa\left(m^{\prime}, 0,0,0\right)+\kappa(m, 0,0,0) \\
& \rho\left(m^{\prime \prime}, 0,0,0\right)=\rho\left(m^{\prime}, 0,0,0\right)+\rho(m, 0,0,0) \\
& \pi\left(m^{\prime \prime}, 0,0,0\right)=\pi\left(m^{\prime}, 0,0,0\right)+\pi(m, 0,0,0) \\
& \lambda\left(0, \tau^{\prime \prime}, 0,0\right)=\lambda\left(0, \tau^{\prime}, 0,0\right)+\lambda(0, \tau, 0,0)-\tau^{\prime} \mu(0, \tau, 0,0) \\
& \mu\left(0, \tau^{\prime \prime}, 0,0\right)=\mu\left(0, \tau^{\prime}, 0,0\right)+\mu(0, \tau, 0,0) \\
& \nu\left(0, \tau^{\prime \prime}, 0,0\right)=\nu\left(0, \tau^{\prime}, 0,0\right)+\nu(0, \tau, 0,0) \\
& \kappa\left(0, \tau^{\prime \prime}, 0,0\right)=\kappa\left(0, \tau^{\prime}, 0,0\right)+\kappa(\tau, 0,0,0) \\
& \rho\left(0, \tau^{\prime \prime}, 0,0\right)=\rho\left(0, \tau^{\prime}, 0,0\right)+\rho(0, \tau, 0,0) \\
& \pi\left(0, \tau^{\prime \prime}, 0,0\right)=\pi\left(0, \tau^{\prime}, 0,0\right)+\pi(0, \tau, 0,0)-\tau^{\prime} \rho(0, \tau, 0,0) \\
& \lambda\left(0,0, a^{\prime \prime}, 0\right)=\lambda\left(0,0, a^{\prime}, 0\right)+\lambda(0,0, a, 0) \\
& \mu\left(0,0, a^{\prime \prime}, 0\right)=\mu\left(0,0, a^{\prime}, 0\right)+\mu(0,0, a, 0) \\
& \nu\left(0,0, a^{\prime \prime}, 0\right)=\nu\left(0,0, a^{\prime}, 0\right)+\nu(0,0, a, 0) \\
& \kappa\left(0,0, a^{\prime \prime}, 0\right)=\kappa\left(0,0, a^{\prime}, 0\right)+\kappa(0,0, a, 0)+a^{\prime} \mu(0,0, a, 0) \\
& \rho\left(0,0, a^{\prime \prime}, 0\right)=\rho\left(0,0, a^{\prime}, 0\right)+\rho(0,0, a, 0)
\end{aligned}
$$




$$
\begin{aligned}
& \pi\left(0,0, a^{\prime \prime}, 0\right)=\pi\left(0,0, a^{\prime}, 0\right)+\pi(0,0, a, 0)-a^{\prime} \nu(0,0, a, 0) \\
& \lambda\left(0,0,0, v^{\prime \prime}\right)=\lambda\left(0,0,0, v^{\prime}\right)+\lambda(0,0,0, v) \\
& \mu\left(0,0,0, v^{\prime \prime}\right)=\mu\left(0,0,0, v^{\prime}\right)+\mu(0,0,0, v) \\
& \nu\left(0,0,0, v^{\prime \prime}\right)=\nu\left(0,0,0, v^{\prime}\right)+\nu(0,0,0, v)-v^{\prime} \mu(0,0,0, v) \\
& \kappa\left(0,0,0, v^{\prime \prime}\right)=\kappa\left(0,0,0, v^{\prime}\right)+\kappa(0,0,0, v)-v^{\prime} \lambda(0,0,0, v) \\
& \rho\left(0,0,0, v^{\prime \prime}\right)=\rho\left(0,0,0, v^{\prime}\right)+\rho(0,0,0, v)+\frac{1}{2} v^{\prime 2} \mu(0,0,0, v)-v^{\prime} \nu(0,0,0, v) \\
& \pi\left(0,0,0, v^{\prime \prime}\right)=\pi\left(0,0,0, v^{\prime}\right)+\pi(0,0,0, v)-\frac{1}{2} v^{\prime 2} \lambda(0,0,0, v)+v^{\prime} \kappa(0,0,0, v)
\end{aligned}
$$

The corresponding solutions read

$$
\begin{aligned}
& \lambda(m, 0,0,0)=a_{1} m \\
& \mu(m, 0,0,0)=a_{2} m \\
& \nu(m, 0,0,0)=a_{3} m \\
& \kappa(m, 0,0,0)=a_{4} m \\
& \rho(m, 0,0,0)=a_{5} m \\
& \pi(m, 0,0,0)=a_{6} m \\
& \lambda(0, \tau, 0,0)=b_{1} \tau-\frac{1}{2} b_{2} \tau^{2} \\
& \mu(0, \tau, 0,0)=b_{2} \tau \\
& \nu(0, \tau, 0,0)=b_{3} \tau \\
& \kappa(0, \tau, 0,0)=b_{4} \tau \\
& \rho(0, \tau, 0,0)=b_{5} \tau \\
& \pi(0, \tau, 0,0)=b_{6} \tau-\frac{1}{2} b_{5} \tau^{2} \\
& \lambda(0,0, a, 0)=c_{1} a \\
& \mu(0,0, a, 0)=c_{2} a \\
& \nu(0,0, a, 0)=c_{3} a \\
& \kappa(0,0, a, 0)=c_{4} a+\frac{1}{2} c_{2} a^{2} \\
& \rho(0,0, a, 0)=c_{5} a
\end{aligned}
$$




$$
\begin{aligned}
& \pi(0,0, a, 0)=c_{6} a-\frac{1}{2} c_{3} a^{2} \\
& \lambda(0,0,0, v)=d_{1} v \\
& \mu(0,0,0, v)=d_{2} v \\
& \nu(0,0,0, v)=d_{3} v-\frac{1}{2} d_{2} v^{2} \\
& \kappa(0,0,0, v)=d_{4} v-\frac{1}{2} d_{1} v^{2} \\
& \rho(0,0,0, v)=d_{5} v+\frac{1}{6} d_{2} v^{3}-\frac{1}{2} d_{3} v^{2} \\
& \pi(0,0,0, v)=d_{6} v-\frac{1}{6} d_{1} v^{3}+\frac{1}{2} d_{4} v^{2} .
\end{aligned}
$$

Now, using Eqs. (29) and (26) we obtain the general form of $\lambda, \mu, \nu, \kappa, \rho$ and $\pi$ (the resulting expressions were reinserted back to Eq. (26) which provided some furhter constraints for parameters):

$$
\begin{aligned}
\lambda(m, \tau, a, v) & =b_{1} \tau+d_{1} v \\
\mu(m, \tau, a, v) & =0 \\
\nu(m, \tau, a, v) & =d_{3} v \\
\kappa(m, \tau, a, v) & =b_{4} \tau-b_{1} a+d_{4} v-\frac{1}{2} d_{1} v^{2} \\
\rho(m, \tau, a, v) & =b_{5} \tau+d_{5} v-\frac{1}{2} d_{3} v^{2} \\
\pi(m, \tau, a, v) & =\left(b_{1}-d_{3}\right) m+b_{6} \tau-\frac{1}{2} b_{5} \tau^{2}+\left(b_{4}+d_{5}\right) a+d_{6} v-\frac{1}{6} d_{1} v^{3} \\
+\frac{1}{2} d_{4} v^{2} & -d_{3} a v-d_{5} v \tau+\frac{1}{2} d_{3} \tau v^{2}
\end{aligned}
$$

The general form of $\eta$ is given by Eqs. (24) and (30). Our next aim is to classify nonequivalent $\eta^{\prime}$ s. As it is well known $\eta$ defines the bialgebra structure on $L$ through

$$
\delta(X)=\left.\frac{d \eta\left(e^{i t x}\right)}{d t}\right|_{t=0}
$$

Simple calculation gives: 


$$
\begin{aligned}
& \delta(H)=-b_{1} H \wedge P-b_{4} H \wedge M-b_{5} M \wedge K-b_{6} M \wedge P \\
& \delta(P)=-b_{1} H \wedge M+\left(b_{4}+d_{5}\right) M \wedge P \\
& \delta(K)=d_{1} H \wedge P+d_{3} P \wedge K+d_{4} H \wedge M+d_{5} M \wedge K+d_{6} M \wedge P \\
& \delta(M)=\left(b_{1}-d_{3}\right) M \wedge P
\end{aligned}
$$

By comparying Eqs. (13) and (32) we get

$$
\begin{aligned}
& a=-b_{1}, e=d_{1}, j=d_{6} \\
& b=-b_{4}, f=d_{3},-(a+f)=b_{1}-d_{3} \\
& c=-b_{5}, g=d_{4}, h-b=b_{4}+d_{5} \\
& d=-b_{6}, h=d_{5},
\end{aligned}
$$

Eq. (33), together with the results of previous section (Table 1) gives us all inequivalent Poisson structures on two-dimensional centrally extended Galilei group. To this end we write out explicitly the general form of Poisson bracket following from Eqs. (10), (20) and (30)

$$
\begin{aligned}
& \{f, g\}=\lambda\left(\frac{\partial f}{\partial \tau} \frac{\partial g}{\partial a}-\frac{\partial f}{\partial a} \frac{\partial g}{\partial \tau}\right)+\kappa\left(\frac{\partial f}{\partial \tau} \frac{\partial g}{\partial m}-\frac{\partial f}{\partial m} \frac{\partial g}{\partial \tau}\right) \\
& +\mu\left(\frac{\partial f}{\partial \tau}\left(\frac{\partial g}{\partial v}-a \frac{\partial g}{\partial m}+\tau \frac{\partial g}{\partial a}\right)-\left(\frac{\partial f}{\partial v}-a \frac{\partial f}{\partial m}+\tau \frac{\partial f}{\partial a}\right) \frac{\partial g}{\partial \tau}\right) \\
& -\nu\left(\frac{\partial f}{\partial a}\left(\frac{\partial g}{\partial v}-a \frac{\partial g}{\partial m}+\tau \frac{\partial g}{\partial a}\right)-\left(\frac{\partial f}{\partial v}-a \frac{\partial f}{\partial m}+\tau \frac{\partial f}{\partial a}\right) \frac{\partial g}{\partial a}\right) \\
& -\rho\left(\frac{\partial f}{\partial m}\left(\frac{\partial g}{\partial v}-a \frac{\partial g}{\partial m}+\tau \frac{\partial g}{\partial a}\right)-\left(\frac{\partial f}{\partial v}-a \frac{\partial f}{\partial m}+\tau \frac{\partial f}{\partial a}\right) \frac{\partial g}{\partial m}\right) \\
& -\pi\left(\frac{\partial f}{\partial m} \frac{\partial g}{\partial a}-\frac{\partial f}{\partial a} \frac{\partial g}{\partial m}\right)
\end{aligned}
$$

In particular, the basic Lie-Poisson brackets read:

$$
\begin{aligned}
& \{v, \tau\}=-\mu=0 \\
& \{v, a\}=\nu=d_{3} v \\
& \{v, m\}=\rho=b_{5} \tau+d_{5} v-\frac{1}{2} d_{3} v^{2}
\end{aligned}
$$




$$
\begin{aligned}
& \{\tau, a\}=\lambda+\tau \mu=b_{1} \tau+d_{1} v \\
& \{\tau, m\}=-a \mu+\kappa=b_{4} \tau-b_{1} a+d_{4} v-\frac{1}{2} d_{1} v^{2} \\
& \{a, m\}=\pi+a \nu+\tau \rho=\frac{1}{2} b_{5} \tau^{2}+\left(b_{1}-d_{3}\right) m+b_{6} \tau+\left(b_{4}+d_{5}\right) a \\
& \quad+d_{6} v-\frac{1}{6} d_{1} v^{3}+\frac{1}{2} d_{4} v^{2}
\end{aligned}
$$

Obviously, there are further constraints on parameters following from Eq. (22) which haven't been used yet. Instead of solving it we impose the Jacobi identities on our Poisson brackets (which is equivalent to solving Eq. (22)). It appears that the additional constaints are, through Eq. (33), equivalent to the ones given by Eq. (14) which provides a further test of the consistency of our results.

\begin{tabular}{|c|c|c|c|c|c|c|}
\hline & $v, a\}$ & $v, m\}$ & $\{\tau, a\}$ & $\{\tau, m\}$ & $\{a, m\}$ & Rem. \\
\hline 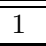 & & & $\overline{\tau_{0}^{2} v}$ & $-\frac{1}{2} \tau_{0}^{2} v^{2}$ & $-\frac{1}{6} \tau_{0}^{2} v^{3}$ & \\
\hline 2 & & & $-\tau_{0}^{2} v$ & $\frac{1}{2} \tau_{0}^{2} v^{2}$ & $\frac{1}{6} \tau_{0}^{2} v^{3}$ & \\
\hline 3 & & $v_{0}^{2} \tau_{0} v$ & & & $v_{0}^{2} \tau_{0} a$ & \\
\hline 4 & & & & $\tau_{0}^{2} v_{0} v$ & $\frac{1}{2} v_{0} \tau_{0}^{2} v^{2}$ & \\
\hline 5 & & & & & $-v_{0}^{3} \tau_{0} \tau$ & \\
\hline 6 & & & & & $v_{0}^{2} \tau_{0}^{2} v$ & \\
\hline 7 & & & & & $-v_{0}^{2} \tau_{0}^{2} v$ & \\
\hline 8 & & $v_{0}^{2} \tau_{0} v$ & & & $v_{0}^{2} \tau_{0} a+v_{0}^{2} \tau_{0}^{2} v$ & \\
\hline 9 & & $v_{0}^{2} \tau_{0} v$ & & & $v_{0}^{2} \tau_{0} a-v_{0}^{2} \tau_{0}^{2} v$ & \\
\hline 10 & & $\bar{v}_{0}^{2} \tau_{0} v$ & $\overline{\tau_{0}{ }^{2} v}$ & $-\frac{1}{2} \tau_{0}^{2} v^{2}$ & $v_{0}^{2} \tau_{0} a-\frac{1}{6} \tau_{0}^{2} v^{3}$ & \\
\hline 11 & & $v_{0}^{2} \tau_{0} v$ & $-\tau_{0}^{2} v$ & $\frac{1}{2} \tau_{0}^{2} v^{2}$ & $v_{0}^{2} \tau_{0} a+\frac{1}{6} \tau_{0}^{2} v^{3}$ & \\
\hline 12 & & & & $\tau_{0}^{2} v_{0} v$ & $\frac{1}{2} v_{0} \tau_{0}^{2} v^{2}-v_{0}^{3} \tau_{0} \tau$ & \\
\hline 13 & $\tau_{0} v_{0} v$ & $-\frac{1}{2} \tau_{0} v_{0} v^{2}$ & & & $\varepsilon v_{0}^{2} \tau_{0}^{2} v-v_{0} \tau_{0} m$ & $\varepsilon \in \mathrm{R}$ \\
\hline 14 & $\tau_{0} v_{0} v$ & $-\frac{1}{2} \tau_{0} v_{0} v^{2}$ & & $\overline{\tau_{0}{ }^{2} v_{0} v}$ & $\varepsilon v_{0}^{2} \tau_{0}^{2} v-v_{0} \tau_{0} m+\frac{1}{2} v_{0} \tau_{0}^{2} v^{2}$ & $\varepsilon \in \mathrm{R}$ \\
\hline 15 & & & $\overline{\tau_{0}}{ }^{2} v$ & $-\frac{1}{2} \tau_{0}^{2} v^{2}$ & $-v_{0}{ }^{3} \tau_{0} \tau-\frac{1}{6} \tau_{0}^{2} v^{3}$ & \\
\hline 16 & & & $-\tau_{0}^{2} v$ & $\frac{1}{2} \tau_{0}^{2} v^{2}$ & $-v_{0}{ }^{3} \tau_{0} \tau+\frac{1}{6} \tau_{0}{ }^{2} v^{3}$ & \\
\hline 17 & & & & $-\tau_{0} v_{0}^{2} \tau$ & $-v_{0}^{2} \tau_{0} a$ & \\
\hline 18 & & & & $-\tau_{0} v_{0}^{2} \tau$ & $-\tau_{0} v_{0}^{3} \tau-\tau_{0} v_{0}^{2} a$ & \\
\hline 19 & & $v_{0}^{2} \tau_{0} v$ & & $-\varepsilon \tau_{0} v_{0}^{2} \tau$ & $(1-\varepsilon) v_{0}^{2} \tau_{0} a$ & $\varepsilon \neq 0$ \\
\hline 20 & & $v_{0}^{2} \tau_{0} v$ & & $\overline{\tau_{0} v_{0}{ }^{2} \tau+\tau_{0}^{2} v_{0} v}$ & $(1+1) v_{0}^{2} \tau_{0} a+\frac{1}{2} v_{0} \tau_{0}^{2} v^{2}$ & \\
\hline 21 & & $-v_{0}^{3} \tau$ & & $-\varepsilon \tau_{0} v_{0}^{2} \tau$ & $-\varepsilon v_{0}^{2} \tau_{0} a-\frac{1}{2} \tau^{2} v_{0}^{3}$ & $\varepsilon \in \mathrm{R}$ \\
\hline 22 & & $-v_{0}^{3} \tau$ & & $-\varepsilon \tau_{0} v_{0}^{2} \tau$ & $-\varepsilon v_{0}^{2} \tau_{0} a-\frac{1}{2} \tau^{2} v_{0}^{3}+v_{0}^{2} \tau_{0}^{2} v$ & $\varepsilon \in \mathrm{R}$ \\
\hline 23 & & $-v_{0}{ }^{3} \tau$ & & $-\varepsilon \tau_{0} v_{0}^{2} \tau$ & $-\varepsilon v_{0}^{2} \tau_{0} a-\frac{1}{2} \tau^{2} v_{0}^{3}-v_{0}^{2} \tau_{0}^{2} v$ & $\varepsilon \in \mathrm{R}$ \\
\hline 24 & & $-v_{0}^{3} \tau$ & & $-\varepsilon \tau_{0} v_{0}^{2} \tau+\tau_{0}^{2} v_{0} v$ & $-\varepsilon v_{0}^{2} \tau_{0} a-\frac{1}{2} \tau^{2} v_{0}{ }^{3}+\frac{1}{2} v_{0} \tau_{0}^{2} v^{2}$ & $\varepsilon \in \mathrm{R}$ \\
\hline 25 & & $-v_{0}^{3} \tau$ & & $-\varepsilon \tau_{0} v_{0}^{2} \tau-\tau_{0}^{2} v_{0} v$ & $-\varepsilon v_{0}^{2} \tau_{0} a-\frac{1}{2} \tau^{2} v_{0}^{3}-\frac{1}{2} v_{0} \tau_{0}^{2} v^{2}$ & $\varepsilon \in \mathrm{R}$ \\
\hline 26 & & & $-v_{0} \tau_{0} \tau$ & $\overline{\tau_{0} v_{0} a}$ & $-v_{0} \tau_{0} m$ & \\
\hline
\end{tabular}

Eqs. (33), (35) and the classification given in Tabele 1 lead us finally to the following classification of nonequivalent Lie-Poisson structures (Table 2)

Table 2 
As far as Table 2 is concerned the following remark is in order. Up to now we were dealing with dimensionless generators and group parameters. In order to take care about proper dimensions we replace our generators by dimensionful ones according to the rules:

$$
H \rightarrow \frac{H}{\tau_{0}}, P \rightarrow \frac{P}{v_{0} \tau_{0}}, K \rightarrow \frac{K}{v_{0}}, M \rightarrow \frac{M}{v_{0}^{2} \tau_{0}}
$$

where $\tau_{0}$ and $v_{0}$ are arbitrary time and velocity units; the group parameters are redefined appropriately. This redefinition has been already taken into account in Table 2

\section{Conclusions}

We have classified all inequivalent bialgebra structures on the centrally extended two-dimensional Galilei algebra and found the corresponding LiePoisson structures on the group. The resulting classification appears to be quite rich and contains 26 inequivalent cases, eigth of them being the coboundary ones. This is in contrast with semisimple case as well as the case of fourdimensional Poincare group where there are only coboundary structures.

\section{Acknowledgment}

The Author acknowledges Prof. P. Kosiński for a careful reading of the manuscript and many helpful suggestion. Special thanks are also due to Prof. S. Giller, Dr. C. Gonera, Prof. P. Maślanka and MSc. E. Kowalczyk for valuable discussion.

\section{References}

[1] F. Bonechi, E. Celeghini, R. Giachetti, E. Sorace, M. Tarlini, J. Phys. 25, 939 (1992).

[2] F. Bonechi, E. Celeghini, R. Giachetti, E. Sorace, M. Tarlini, Quantum Galilei Group as Symetry of Magnons, hep-th/9203048. 
[3] J.A. de Azcarraga, J.G. Perez Bueno, J.Phys A 29, 6353 (1996).

[4] J.A. de Azcarraga, J.G. Perez Bueno, J. Math. Phys. 36, 6879 (1995).

[5] A. Ballesteros, F.J. Herranz, M.A. de Olmo, M.Santader, J. Phys. A27, 1283 (1994).

[6] J. Lukierski, M. Ruegg, V.N. Tolstoy, in:, Quantum Groups. Formalism and Applications, Proc. of XXX Karpacz Winter School of Theoretical Physics, eds. J. Lukierski, Z. Popowicz, J. Sobczyk, PWN(1995) and references therein.

[7] J. Lukierski, A. Nowicki, H. Ruegg, Phys. Lett. B293, 344 (1961).

[8] E. Kowalczyk, Acta Phys. Pol. B28, 1893 (1997).

[9] M. Levy-Leblond, J. Math. Phys. 4, 776 (1961).

[10] J. Sobczyk, J. Phys. A29, 2887 (1996). 